\title{
The effect of hematocrit on solid-phase microextraction
}

\author{
Nathaly Reyes-Garcés, Md. Nazmul Alam, Janusz Pawliszyn*
}

Department of Chemistry, University of Waterloo, Waterloo, Ontario, Canada N2L 3G1

* Corresponding author: Tel.: +1 519888 4641; fax: +1 519746 0435. E-mail address:

Janusz@uwaterloo.ca (J. Pawliszyn).

The final publication is available at Elsevier via https://doi.org/10.1016/j.aca.2017.11.014 @ 2017. This manuscript version is made available under the CC-BY-NC-ND 4.0 license

http://creativecommons.org/licenses/by-nc-nd/4.0/ 


\section{Abstract}

Solid-phase microextraction (SPME) is an approach to sample preparation that has demonstrated its appropriateness for isolating/enriching analytes present in complex biofluids with minimum sample pre-treatment. Several prior in vitro and in vivo studies have used SPME to monitor the concentrations of various drugs and metabolites in blood samples. In this work, we present the results of an investigation into how various levels of hematocrit $(\mathrm{Hct})$ affect SPME recoveries. The matrices for this study consisted of whole blood samples that had been adjusted at three different Hct levels $(20 \%, 45 \%$, and $70 \%)$, and the selected model compounds were drugs with different physicochemical characteristics (log $P$ range from 0.33 to 6.36 ). In addition, two experimental setups were employed to conduct the extractions: hydrophilic lipophilic balanced (HLB) coated SPME devices (HLB-D) at $1500 \mathrm{rpm}$ (vortex agitation), and mixed mode SPME fibres (MM-F) at $400 \mathrm{rpm}$ (orbital shaking agitation). Our results demonstrated that the Hct effect in SPME is dependent on the analytes of interest, and that different outcomes can be attained by altering experimental conditions, such as coating type, convection, and extraction time. Interestingly, a target compound's relative affinity for the matrix components and for the coating material proved to be one of the main factors that determine the final effect that different erythrocyte levels have on SPME recoveries. Finally, although the Hct content affects each analyte differently and the final Hct effect depends on the experimental parameters, matrix variability can be corrected by using appropriate internal standards, thereby resulting in correct quantification. 
Key words: solid-phase microextraction, SPME, blood, red blood cells, hematocrit effect, sample preparation

Abbreviations: Solid-phase microextraction (SPME), hematocrit (Hct), hydrophilic lipophilic balanced (HLB), hydrophilic lipophilic balanced coated SPME devices (HLB-D), mixed mode fibres (MM-F), dried blood spots (DBS)

\section{Highlights:}

- In this work an investigation into how the hematocrit (Hct) affects SPME recoveries is presented.

- Our results demonstrated that the Hct effect in SPME is dependent on the analytes, and that different outcomes can be attained by altering experimental conditions.

- The relative affinity of a target compound for the matrix components and for the coating proved to be a critical factor that determines the final Hct effect on SPME.

- By using appropriate internal standards, variable SPME recoveries due to different Hct levels can be corrected. 


\section{Introduction}

Many bioanalytical studies and clinical tests rely on the quantitative determination of small molecules in whole blood samples. Given the complexity of this type of matrix, developing simple analytical workflows that can provide satisfactory results while remaining compliant with health regulations can be a challenging undertaking. Whole blood is comprised of two main components: plasma and blood cells. Plasma has a relatively simpler composition than whole blood, which makes it a commonly-used matrix for many routine bioanalytical determinations, the most representative case of which being the therapeutic monitoring of multiple drugs. The second main component of whole blood is the cells, which can be classified into three main types: red blood cells (erythrocytes, 96 \%), white blood cells, and platelets [1]. Hematocrit (Hct) is a term that refers to the fraction of blood volume made up of red blood cells, and its typical values are within the ranges of $40-50 \%$ for men, and $36-44 \%$ for women [2]. However, Hct levels can fall outside of these ranges in particular cases, such as individuals living at high altitudes, newborns, or anemic people [3]. Although plasma and serum are the specimens of choice for several standardized analytical procedures, the direct analysis of whole blood samples is required in certain instances (e.g. in forensic toxicology, in the determination of immunosuppressive drugs, among others). Hence, understanding and evaluating Hct's effect on whole blood samples analysis should always be a matter of consideration.

Numerous sample preparation methodologies for analyzing small compounds ( $<1500 \mathrm{Da})$ in whole blood samples using liquid chromatography coupled to mass spectrometry have been reported in the literature [4-11]. Among such approaches, liquid-liquid extraction (LLE) and 
protein precipitation (PP) - which, in some cases is followed by cleaning steps involving online or offline solid-phase extraction-are perhaps the most commonly used strategies. For substances that display a high affinity for red blood cells, the use of additives that induce hemolysis and the release of compounds from erythrocyte membranes (e.g. zinc sulfate) have been reported as effective means of normalizing the sample matrix composition [12-14]. Nonetheless, the use of appropriate internal standards is always recommended in order to ensure satisfactory method accuracy and precision. Due to the highly-invasive nature of traditional blood collection, alternative blood micro sampling strategies have been garnering much interest. For instance, dried blood spots (DBS), which is a well-established sampling and whole-blood-analysis methodology, has been broadly reported in several applications due to its low invasiveness $[7,9,15-18]$. In DBS, a small drop of whole blood is placed on filter paper, allowed to dry, and subsequently analyzed by desorbing fixed-diameter discs punched out from the dried spots. Prior studies have found that Hct's effect on DBS analysis is mostly associated with the variable spreading pattern that blood samples exhibit when spotted onto DBS cards $[2,3,7]$; the viscosity of blood samples is directly related to their Hct values, and samples with higher Hct values produce smaller spot radii. The best strategy for overcoming Hct's impact in relation to variable blood spreading is to analyze whole spots of volumetrically-applied blood $[3,7,19]$. The incorporation of appropriate internal standards via spraying prior to DBS extraction has proven to be a feasible way of mitigating the variations related to matrix effects or recoveries $[20,21]$. Another absorptive-based approach recently introduced to overcome the Hct issues associated with DBS is volumetric absorptive microsampling (VAMS) [8], which uses an absorptive polymeric material immobilized on a pipette tip to collect fixed volumes of blood via wicking. Several studies 
have demonstrated that VAMS devices are able to collect reproducible blood volumes independent of their Hct levels $[8,22,23]$. However, some issues with VAMS have also been reported, particularly in relation to bias in the determination of blood concentrations, low recoveries at high Hct levels, and interoperator variability $[22,23]$. Further evaluation of VAMS is still required as it is still a relatively new microsampling approach.

Solid-phase microextraction (SPME) is a non-exhaustive extraction technique that uses a matrixcompatible coating structure, which enables the analysis of complex samples, such as plasma and even whole blood. In fact, the literature contains several prior studies that document SPME's usefulness as a sample preparation approach for such matrices prior to LC-MS or direct MS instrumental analysis [11,24-28]. Interestingly, SPME's applicability is not only limited to ex vivo determinations; indeed, several studies have documented its suitability for in vivo blood analysis in different animal models [29-36]. In regards to the principle of SPME, it is worth mentioning that the extraction and pre-concentration of analytes occurs by directly exposing a defined amount of a biocompatible extraction phase to the sample matrix for a given period of time. The amount of analyte collected during the extraction process is proportional to the analyte's concentration and depends on the analyte's affinity for the biocompatible coating and for the sample matrix at the extraction conditions. Considering that the SPME matrix compatible coating is highly selective for small compounds and that a washing step is carried out after the extraction, the presence of proteins, salts, and other interferences that could affect the instrumental analysis is easily avoided. In regards to the extraction mechanism, it is worth emphasizing that, in SPME, the uptake of analytes from the sample media to the coating material happens via free analyte concentration [37]. Therefore, if we consider a complex system, such as blood samples, where 
multiple co-existing phases can display an affinity for a given target compound, the amount of analyte extracted by the SPME probe will be correlated to the free concentration of analyte at the end of the extraction. As a matter of fact, parameters such as lipophilicity, charge, molecular weight, diffusion coefficient, and structural moieties govern the distribution of any compound into different blood compartments [38]. Likewise, such parameters strongly determine the affinity that a target analyte exhibits for a particular SPME coating. In addition to the analyte's physicochemical characteristics, there are other factors that play an important role in the microextraction process; for example, extraction time, extraction phase characteristics, temperature, and convection conditions all determine the final recovery provided by an SPME device. Given that red blood cells comprise a significant portion of a whole blood sample, this work aims to investigate how varying Hct levels affect the amount of analyte extracted by SPME devices. For this purpose, ten drugs with different polarities and physicochemical properties were chosen as model compounds, and blood samples with three different Hct levels (20\%, 45\%, and $70 \%)$ were selected as sample matrices. The effects of the coating type, sample convection, and extraction time were also taken into consideration.

\section{Experimental}

\subsection{Materials and supplies}

Methamphetamine, stanozolol, codeine, codeine-d3, ( \pm )11-nor-9-carboxy- $\triangle 9-T H C$ (THCCOOH), ( \pm 11-nor-9-carboxy- $\triangle$ 9-THC-d3 (THCCOOH-d3), oxycodone-d3, testosterone-d3, cannabidiol-d3 and methadone-d3 standards were obtained from Cerilliant Corporation (Round Rock, TX, USA). Nikethamide, propranolol, metoprolol, clenbuterol, exemestane, and salbutamol were 
purchased from Millipore Sigma (Oakville, ON, Canada), and salbutamol-d3 was purchased from CDN Isotopes (Pointe Claire, Quebec, Canada). Sodium chloride, potassium chloride, potassium phosphate monobasic, sodium phosphate dibasic, and formic acid were also purchased from Millipore Sigma (Oakville, ON, Canada). LC-MS grade acetonitrile, methanol, and water were obtained from Fischer Scientific.

Biocompatible SPME fibres (MM-F) coated with a mixed-mode extraction phase (C18 and benzene sulfonic acid functionalities, $1.5 \mathrm{~cm}$ coating length, $45 \mu \mathrm{m}$ coating thickness, and a total diameter of $300 \mu \mathrm{m}$ ) were generously provided by Millipore Sigma (Bellefonte, PA, USA). SPME devices prepared on a plastic support with a hydrophilic lipophilic balanced (HLB) extraction phase (HLB-D) ( $2 \mathrm{~cm}$ coating length, $150 \mu \mathrm{m}$ coating thickness, and $2 \mathrm{~mm}$ total diameter) were made as described in [25].

Blood samples were purchased from Bioreclamation IVT (Westbury, New York, USA). These samples were from healthy donors (with K2-EDTA as anticoagulant), and had Hct levels that had been adjusted to $20 \%, 45 \%$, and $70 \%$.

\subsection{Working solutions}

A stock methanolic solution (20 $\left.\mathrm{g} \mathrm{m} \mathrm{m}^{-1}\right)$ containing all analytes (nikethamide, salbutamol, codeine, metoprolol, methamphetamine, clenbuterol, exemestane, propranolol, stanozolol and THCCOOH) was prepared and further dilution was done as required. A stock solution ( $\left.8 \mu \mathrm{g} \mathrm{mL}^{-1}\right)$ containing different deuterated compounds as internal standards was prepared in methanol.

\subsection{SPME procedure}


Prior to the experiments, the blood samples with adjusted Hct content were spiked with stock methanolic solutions containing all the analytes, while ensuring that the organic solvent content was kept below $1 \%$ in all cases. For the construction of the calibration curves, the spiked blood samples were pre-incubated for at least 1 hour at constant agitation; for the determination of the extraction time profiles, blood samples incubation was allowed overnight. Before the extractions, MM-F and HLB-D were first pre-conditioned in a 1:1 (v/v) methanol:water solution for 30 min using vortex agitation at $1500 \mathrm{rpm}$. Subsequently, all devices were rinsed in ultrapure water for $10 \mathrm{~s}$ to remove any remaining organic solvent from the coating surface. In order to guarantee a stable sample $\mathrm{pH}$ (close to physiological $\mathrm{pH}$ ) over the entire extraction process, 1080 $\mu \mathrm{L}$ of spiked blood aliquots were mixed with $120 \mu \mathrm{L}$ of $1 \mathrm{M}$ phosphate buffer adjusted at a pH of 7. Furthermore, $15 \mu \mathrm{L}$ of internal standard solution were added to the samples used for the calibration curve experiments. The extractions were then carried out by immersing both types of SPME devices into the sample matrix for a pre-set period of time at constant agitation conditions. To construct the calibration curves, the extraction time was set at $90 \mathrm{~min}$, the calibration range was set between 5 and $100 \mathrm{ng} \mathrm{mL}^{-1}$ for all target analyte, and four calibration curve replicates were constructed on two consecutive days. Time points of 15, 30, 60, 90, 120 and 180 min were selected for the extraction time profiles, and all the extractions were conducted in triplicates. The agitation parameters were set as follows: vortex agitation was set at $1500 \mathrm{rpm}$ for the HLBD, and orbital shaking agitation was set at $400 \mathrm{rpm}$ for the MM-F. Once the extraction step was completed, three consecutive rinsing steps in water ( $5 \mathrm{~s}$ each) were performed following the procedure reported by Reyes-Garces et al [25]. Finally, desorption of the devices was carried out for $20 \mathrm{~min}$ at $1500 \mathrm{rpm}$ in 300 and $600 \mu \mathrm{L}$ of desorption solution (4:1 methanol:acetonitrile) for 
MM-F and HLB-D, respectively. To determine the amount of analyte extracted by the SPME devices in each experiment, an instrumental calibration curve (from 0.1 to $150 \mathrm{ng} \mathrm{mL}^{-1}$ ) was prepared using the same solvent composition that was used for the desorption solution.

\subsection{LC-MS/MS conditions and data processing}

All the collected extracts and the instrumental calibration curve were run using the LC-MS/MS conditions reported in [25]. Briefly, the LC-MS/MS system was comprised of an Accela autosampler, an Accela pump, and a TSQ vantage triple quadrupole mass spectrometer with a heated electrospray ionization source operating in positive mode (Thermo Scientific, San Jose, USA). Chromatographic separation was attained using a Kinetex pentafluorophenyl core shell column $(1.7 \mu \mathrm{m}, 2.1 \mathrm{~mm} \times 10 \mathrm{~mm})$ that had been connected to a PFP security guard ultracartridge (Phenomenex, Torrance, CA, USA), and LC separation was achieved using a mobile phase system consisting of $0.1 \%$ formic acid (A), acetonitrile with $0.1 \%$ formic acid (B), and methanol with $0.1 \%$ formic acid (C). The gradient elution conditions were set as follows: $A, B$, and C were held at $90 \%$, $5 \%$, and $5 \%$, respectively, for $0.5 \mathrm{~min}$; B and C were then increased linearly to $50 \%$ over the course of $6.5 \mathrm{~min}$; C was then increased to $75 \%$ and B was decreased to $25 \%$ over the course of $5 \mathrm{~min}$ and held for $3.5 \mathrm{~min}$. Finally, the column was kept at the initial gradient composition for 2 $\min$. The column temperature was kept at $35^{\circ} \mathrm{C}$, the total run time was $17.5 \mathrm{~min}$, and the column flow was set at $0.3 \mathrm{~mL} \mathrm{~min}^{-1}$. Samples were stored in the autosampler at $5{ }^{\circ} \mathrm{C}$, and the injection volume was $10 \mu \mathrm{L}$. MS analysis was conducted using selective reaction monitoring (SRM) mode, and the conditions of each compound were optimized by directly infusing the standards. Table 1 summarizes information corresponding to SRM transitions of the model compounds The 
following transitions were selected for the deuterated compounds that were employed as internal standards: salbutamol-d3: $243.16 \rightarrow 151.12$; codeine-d3: $303.15 \rightarrow 165.10$; oxycodoned3: $319.12 \rightarrow$ 259.15; methadone-d3: $313.21 \rightarrow$ 105.10; testosterone-d3: $292.25 \rightarrow$ 97.14; and THCCOOH-d3: $348.16 \rightarrow 302.28$. Other parameters were set as follows: spray voltage $=1300 \mathrm{~V}$; vaporizer temperature $=275^{\circ} \mathrm{C}$; sheath gas $=45$ units; auxiliary gas $=30$ units; and capillary temperature $=280^{\circ} \mathrm{C}$.

\subsection{Data analysis}

Xcalibur software (2.0.7 SP1) was employed for data acquisition and processing. For the purpose of this study, the slopes of the calibration curves constructed for each compound with different Hct levels were statistically compared. Statistical analysis was carried out using one-way ANOVA, one-tail t-test (equal and unequal variances), and relative standard deviations (RSDs).

\section{Results and discussion}

In order to elucidate the effect that various Hct levels can have on SPME recovery rates, an analyte's affinity for the main blood components (aqueous phase, proteins, and red blood cells) should be taken into consideration. For this reason, analytes with distinct physicochemical 
properties were selected for this work because they can be expected to display variable affinities for blood constituents (Table 1). In addition to using various model compounds, two experimental setups were employed: MM-F at $400 \mathrm{rpm}$ and HLB-D at $1500 \mathrm{rpm}$. Although both types of SPME devices have been documented as being suitable and biocompatible for the analysis of whole blood samples $[25,32,39]$, the attachment of macromolecules is usually observed in the case of MM-F under aggressive agitation conditions and long extraction times. Consequently, an orbital shaker operating at $400 \mathrm{rpm}$ was chosen as the agitation setup for MMF extractions. In the case of HLB-D, satisfactory results for whole blood samples analysis were already found when using vortex agitation at $1500 \mathrm{rpm}$; therefore, the same parameters were kept [25]. Although high agitation speeds such as $1500 \mathrm{rpm}$ are likely to induce lysis in red blood cells-and therefore changes in the Hct content during the extraction [40] - the main purpose of the evaluation was to assess the overall effect of different red blood cell contents on SPME extractions under typical experimental conditions.

Table 2 presents the results corresponding to the calculated slopes for each of the model analytes in blood samples with different Hct levels as determined by the two different experimental setups. It is worth emphasizing that slope calculation was selected as parameter for investigating Hct effect because it is a well-accepted approach for assessing relative matrix effects [41]. As shown in Table 2, different outcomes were found depending on the analyte and the experimental conditions; therefore, in order to simplify things, we classified the observations into four different cases: no Hct effect at any of the experimental conditions (Case I), Hct effect only when MM-F were used (Case II), Hct effect in both MM-F and HLB-D experiments (Case III), and Hct effect when HLB-D were used (Case IV). 
In the first case (Case I), no statistically significant Hct effect was observed for any of the study conditions (MM-F at $400 \mathrm{rpm}$ and HLB-D at $1500 \mathrm{rpm}$ ). Interestingly, salbutamol, nikethamide, codeine, and methamphetamine, which are all characterized as being relatively polar and/or for having low protein-binding affinities, were categorized in this first group. In order to explain the obtained results, parameters such as the polarity and affinity of an analyte for the matrix components and the extraction phases should be taken into account. With regards to an analyte's polarity, it is important to emphasize that highly-polar compounds typically display shorter equilibration times due to their low affinities for SPME coatings (low $K$ values). This is confirmed by Equation 1, where the equilibration time in SPME is expressed as a function of the boundary layer thickness $\left(\delta_{S}\right)$, the distribution constant $(K)$, the thickness of the SPME coating $(L)$, the diffusion coefficient of the analyte in the sample matrix $\left(D_{s}\right)$, and the coating capacity $\left(\Gamma_{\max }\right)$ [42].

$$
t_{e q} \approx t_{95 \%}=\frac{\delta_{s} K L}{D_{S} \Gamma_{\max }}
$$

In this context, it is worth mentioning that, when extractions are performed at conditions close to equilibrium, the amount of analyte collected is mostly determined by the analyte's distribution constant. Conversely, when the extraction process is interrupted at an earlier stage, the diffusion of the analytes in the sample matrix plays a more important role. Since 90 min was chosen as the extraction time for the Hct effect assessment, it was relevant for the interpretation of the results to determine at what point of the extraction process the comparison was being conducted. As shown in Figure $1 \mathrm{~A}$, a relatively polar compound like salbutamol was close to reaching equilibrium after 90 min of extraction using MM-F at $400 \mathrm{rpm}$. This means that variations in the 
analyte's diffusivity due to differences in the matrix viscosity will not have an important effect on the analyte's uptake. Moreover, as can be seen in the same plot, minimal differences were found in the profiles constructed at the three Hct levels. Considering salbutamol's high polarity and low protein binding, variable levels of matrix constituents are not expected to greatly affect SPME recoveries as a result of decreasing the analyte's free concentration. In the case of HLB-D, a slightly different situation was observed for the same compound. As can be seen in Figure 1B, although there is an overlap in the constructed time profiles for blood samples adjusted at different Hct levels, a plateau in the extracted amounts of salbutamol was not achieved, even after 180 min of extraction at high-speed agitation. These results can be explained based on HLB's much higher affinity for polar analytes and the thicker HLB-D coatings that permit the extraction of much larger amounts of analyte. Under such experimental conditions, the stronger affinity that HLB-D displays for the target analytes-compared to the one exhibited by the matrix constituents-will contribute to lessening the differences that can occur in SPME recoveries due to variations in the matrix composition. It is also noteworthy that the convection parameters in the HLB-D experiments were significantly higher than those in the MM-F extractions. Faster agitation conditions allow for a decrease in the thickness of the boundary layer, which in turn accelerates the mass transfer process occurring from the sample matrix to the SPME coating. Given this principle, the combination of improved convection conditions and HLB-D's strong extraction capacity will result in a reduced Hct effect in the extraction of analytes that do not show a specific affinity for erythrocytes. In the case of compounds such as methamphetamine and codeine, which are less polar than salbutamol and nikethamide but still meet the conditions to be classified as Case I, it is noteworthy that the slopes of the calibrations curves constructed 
with MM-F showed higher RSD values $(9.5 \%$ and $8 \%$ ) than those obtained with HLB-D (1\% and 0.1\%). In fact, when MM-F was used, the slopes for methamphetamine and codeine were statistically higher in blood samples adjusted at $20 \% \mathrm{Hct}$ than for blood samples adjusted at 70 $\%$ Hct (methamphetamine ( $20 \%$ vs $70 \%$ Hct): one-sample $t(6)=3.52$, p-value $<0.01$; codeine $(20 \%$ vs $70 \% \mathrm{Hct}$ ): one-sample $\mathrm{t}(6)=4.42, \mathrm{p}<0.01)$. Since these compounds are slightly hydrophobic, and considering the fact that codeine binds to the red blood cell plasma membrane, the presence of variable levels of matrix components is likely to alter the concentration of analytes available for SPME extractions [43]. Interestingly, this effect becomes more pronounced when devices with weaker affinities and lower extraction capacities, such as MM-F, are employed for extraction. In light of the discussed results, it is clear that the affinity of target analytes for the coating and the matrix components has a highly-significant effect on the final SPME recoveries when analyzing blood samples with different Hct levels.

In order to explain the experimental findings corresponding to Case II, the same rationale regarding various analytes' affinities for coatings and for matrix constituents should be taken into consideration. As can be seen in Table 2, Case II analytes had different outcomes that were dependent on the SPME experimental parameters. On the one hand, a pronounced decrease in the slopes as a function of erythrocyte levels was observed in the experiments conducted with MM-F; on the other hand, the statistical comparison of the slopes showed no Hct effect for extractions carried out with HLB-D (propranolol: $F(2,9)=0.232$, $p$-value=0.80; metoprolol: $F(2,9)=0.243, p$-value $=0.79$; clenbuterol: $F(2,9)=1.031, p$-value $=0.40)$. The lack of observed differences when HLB was used can be attributed to the high convection conditions, as well as the fact that, as was discussed in relation to Case I, HLB-D has a much higher affinity for the target 
analytes than MM-F and matrix components. Moreover, since the Case II analytes are overall less polar than those in Case I, they can be expected to have a higher degree of interaction with matrix components (lower free concentrations) and larger $K$ values, and therefore longer equilibration times (Equation 1). Figure $1 \mathrm{C}$ presents the extraction time profile constructed for propranolol using MM-F at $400 \mathrm{rpm}$. As can be seen, the recoveries corresponding to the profile in $20 \% \mathrm{Hct}$ blood samples are considerably higher than those obtained for the other two Hct levels. This outcome can be explained based on the fact that higher concentrations of matrix componentsin this case, red blood cells-lead to lower concentrations of propranolol available for SPME extraction; thus, lower amounts of propranolol are collected at $70 \%$ Hct. Given that MM-F lacks sufficient extraction capability for inducing the release of bound analytes in order to further enhance the extraction recoveries, such experimental conditions tend to exhibit a significant Hct effect. Moreover, taking into account that the extraction process is still close to the linear regime at $90 \mathrm{~min}$, it is possible that differences in the analyte's diffusivity as a function of matrix viscosity could be partly responsible for variations in the uptake rate [44]. Although equilibrium is far from being reached at 90 min in HLB-D (Figure 1D), its high affinity for propranolol and the high convection conditions largely mitigate any effect that variable Hct levels can have on the final recoveries. Indeed, HLB's high capacity for adsorption is able to deplete the analyte's free concentration and induce the release of bound analytes back to their free forms, thereby increasing the total recovery. While possible changes in the matrix composition due to vigorous agitation [40] should be considered when explaining the different recoveries obtained using the two experimental setups, the results that will be presented in the next case clearly indicate that red blood cells remain a significant compartment after 90 min of vortex agitation. 
In the third case noted in this study (Case III), a similar Hct effect for both MM-F and HLB-D was found; that is, the amount of analyte extracted decreased in proportion to the blood erythrocyte levels. This particular behavior was observed for exemestane, a relatively non-polar steroidal compound (Figures $1 \mathrm{E}$ and $1 \mathrm{~F}$ ). As shown in Table 2, extractions conducted with both MM-F and HLB-D produced higher calibration curve slopes for $20 \%$ Hct blood samples than for the ones calculated at 70\% (MM-F (20\% vs 70\% Hct): one-sample $t(5)=7.25, p<0.01 ;$ HLB-D (20\% vs 70\% $\mathrm{Hct})$ : one-sample $\mathrm{t}(4)=3.37, \mathrm{p}<0.05)$. In the case of $M M-F$, a more pronounced variation was observed in the slopes of the calibration curves constructed at the different Hct levels due to the previously described effects of lower coating affinity and slower agitation. To the best of our knowledge, there is no information in the literature regarding the partitioning of exemestane into red blood cells. In fact, stanozolol, another steroidal compound that was used as a model analyte in this study, did not exhibit the same Hct effect. In order to confirm that exemestane was being partitioned into the erythrocytes, the recoveries of extractions from $70 \%$ Hct blood samples and fully-hemolyzed $70 \%$ Hct blood samples were compared using HLB-D. Complete blood hemolysis was achieved by storing pre-spiked $70 \%$ Hct blood samples for two hours at -80 ${ }^{\circ} \mathrm{C}$ (i.e. freeze and thaw). As shown in Figure 2, the recovery of exemestane increased by approximately $60 \%$ when extracted from hemolyzed blood; this confirms that this drug is being partitioned into the red blood cells. To verify that the changes observed in exemestane recoveries were due to blood hemolysis and not due to ionization effects (suppression/enhancement), absolute matrix effects were assessed in $70 \%$ Hct blood samples (hemolyzed and non-hemolyzed) according to the procedure proposed by by Matuszewski et al (Figure 3) [41]. Based on these results, it is evident that exemestane's affinity for the matrix is much higher than that of other 
model analytes categorized in the previous cases such that even pronounced differences are observed when HLB-D are used. Therefore, the use of an internal standard that displays the same affinity for red blood cells is crucial to ensuring the successful analysis of exemestane in blood samples obtained from different patients.

Similar to Case II, two different trends that were dependent on the SPME experimental conditions were observed in Case IV. Interestingly, the model compounds that were categorized within this last case are highly lipophilic and capable of significant protein binding. The slopes for these analytes were significantly higher at $70 \%$ Hct content when the extractions were carried out using HLB-D at 1500 rpm (THCCOOH (20\% vs 70\% Hct): one-sample t(5)=-8.58, p<0.01; stanozolol (20\% vs $70 \% \mathrm{Hct}$ ): one-sample $t(6)=-4.89, p<0.01$ ) (Table 2$)$. On the contrary, no statistical differences in the slopes were observed for the calibration curves constructed with MM-F (THCCOOH: $F(2,8)=1.371, p$-value $=0.31$; stanozolol: $F(2,9)=1.539, p$-value $=0.27)$. One of the model compounds that exhibited this behavior was $\mathrm{THCCOOH}$, the main secondary metabolite of THC. THCCOOH is a considerably non-polar analyte (logP 6.38) with $92.0 \pm 8.7 \%$ protein binding [45]. In addition, according to previous studies, THCCOOH's distribution into red blood cells is restricted $[45,46]$. Based on this behavior, the concentration of $\mathrm{THCCOOH}$ in plasma will be significantly higher than its concentration in whole blood (blood-to-plasma ratio $=0.65$ )[45]. Therefore, if we take into account that the volume of plasma is substantially less at $70 \%$ Hct than at $20 \%$ and $45 \% \mathrm{Hct}$, we can anticipate that a higher free analyte concentration will be available for SPME extractions at $70 \%$ Hct than at the other two Hct levels. This trend is reflected in the slopes for THCCOOH that were obtained with HLB-D (Table 2), and in its extraction time profile (Figure $1 \mathrm{H}$ ). A similar behavior was also observed in the slopes for stanozolol that were 
constructed under the same conditions. Stanozolol is also known for being highly lipophilic (logP 4.43), but, to the best of our knowledge, there have been no prior studies that have analyzed its distribution in different blood components. With respect to the results obtained using MM-F, we hypothesize that the concentration available for SPME extractions, the convection conditions, and the diffusion of analytes all played an important role in the final outcome. As can be seen in Figure 1G, the $\mathrm{THCCOOH}$ uptake in $\mathrm{MM}-\mathrm{F}$ is still in its linear regime at 90 min due to the slow convection and the compound's high affinity for the coating. Hence, the analytes' diffusion has a more significant effect on the extraction process than it does in cases where extractions are closer to equilibrium. In addition, it is important to consider that the flux of analyte from the sample matrix towards the coating (or analyte uptake) is proportional to the concentration gradient between the coating surface and the sample. Since a higher concentration of analyte is available for SPME extractions at $70 \% \mathrm{Hct}$, a higher uptake rate can be expected due to the concentration gradient. Based on this information, the estimated slopes for MM-F represent a combination of both the variation in analyte diffusion at different sample viscosities, and the changes in analyte uptake rates due to the concentrations available for SPME extractions at different Hct levels.

As has been discussed so far, the Hct effect in SPME is completely dependent on the analytes of interest, and different outcomes can be obtained by changing the experimental conditions. Parameters such as an analyte's physicochemical properties (lipophilicity, molecular weight, diffusivity, etc), distribution constants, convection conditions, and, to some extent, sample viscocity are all important in determining how Hct differences affect SPME recoveries. Nevertheless, as in any other sample preparation approach, the use of internal standards 
represents the best way to account for any matrix variability. Table 3 presents the slopes after internal standard correction for those cases where F>Fcrit. Among seven deuterated drugs tested as internal standards (please refer to section 2.1), only those able to provide acceptable corrections were selected. Unfortunately, none of the available deuterated standards exhibited the same affinity for red blood cells shown by exemestane; as such, we could not provide the corrected data for that compound. As can be seen in Table 3, the RSD values corresponding to the corrected slopes of those analytes that were significantly affected by variable red blood cell content were rewarding. Interestingly, compounds that were not necessarily deuterated analogues of the target analytes were able to provide adequate correction. Several studies in which SPME was used to analyze multiple substances in biofluids have described the use of a small set of internal standards as a practical and cost-effective strategy for correcting all of the target analytes $[24,25,47]$. However, as has been noted above, the behavior of a given analyte in a specific matrix depends on several factors, and a proper assessment of an internal standard's suitability at various Hct levels is required when dealing with real blood samples. Indeed, although a given internal standard can effectively correct for extraction variations in a single blood lot, this does not necessarily mean that the same internal standard will properly account for varying Hct content.

Further investigation should be conducted into alternative strategies for overcoming the Hct effect observed in SPME. These strategies may focus on full red blood cell lysis via a combination of using additives (e.g. zinc sulfate or organic solvents) or freezing and the addition of appropriate internal standards, preferably deuterated analogues. In the case of in vivo blood sampling, special 
attention should be given to the Hct effect when external calibration curves are used for quantitation, as no internal standard can be introduced into the samples.

\section{Conclusions}

In this study, the effect of varying levels of Hct on SPME recoveries was investigated by using distinct model compounds and two different experimental setups. As expected, the observed effect was dependent on the analyte's characteristics and the extraction parameters. For instance, variable Hct levels did not show any effect on the recovery of polar compounds with low protein affinities (e.g. salbutamol) under any of the study conditions. However, the extraction of more hydrophobic analytes, such propranolol, was strongly affected by the red blood cell content only when MM-F was used at $400 \mathrm{rpm}$. Similarly, for compounds such as methamphetamine, codeine, propranolol, metoprolol and clenbuterol, the use of HLB-D with high convection conditions produced negligible differences in the SPME recoveries from blood samples adjusted at various Hct levels. This demonstrated that an analyte's relative affinity for the matrix and the extraction phase is critical when matrices with varying compositions are analyzed. Other model analytes, such as exemestane and $\mathrm{THCCOOH}$, exhibited a negative and positive correlation, respectively, between the SPME recoveries and the Hct content due to their opposite affinity for red blood cells. Lastly, despite variations in analyte recovery due to different erythrocyte contents, it was possible to correct for relative matrix effects by using appropriate internal standards. Overall, since each analyte can display different Hct effects, variable red blood 
cell levels within the final experimental conditions should be always properly assessed when analyzing whole blood samples with SPME.

\section{Acknowledgment}

This study was financed by the Natural Sciences and Engineering Research Council (NSERC) of Canada. The authors of this work would like to thank Dr. Barbara Bojko for her suggestions at the beginning of this study.

\section{References}

[1] J. Schaller, S.A. Gerber, U. Kampfer, S. Lejon, C. Trachsel, Human Blood Plasma Proteins: Structure and Function, John Wiley \& Sons, 2008.

[2] P. Denniff, N. Spooner, The effect of hematocrit on assay bias when using DBS samples for the quantitative bioanalysis of drugs, Bioanalysis. 2 (2010) 1385-1395. doi:10.4155/bio.10.103.

[3] P.M. De Kesel, N. Sadones, S. Capiau, W.E. Lambert, C.P. Stove, Hemato-critical issues in quantitative analysis of dried blood spots: challenges and solutions, Bioanalysis. 5 (2013) 2023-2041. doi:10.4155/bio.13.156.

[4] L. Nováková, H. Vlcková, A review of current trends and advances in modern bio- 
analytical methods: chromatography and sample preparation., Anal. Chim. Acta. 656 (2009) 8-35. doi:10.1016/j.aca.2009.10.004.

[5] H. Gika, G. Theodoridis, Sample preparation prior to the LC-MS-based metabolomics/metabonomics of blood-derived samples, Bioanalysis. 3 (2011) 16471661. http://www.future-science.com/doi/pdfplus/10.4155/bio.11.122 (accessed March 16, 2017).

[6] D. Grote-Koska, S. Czajkowski, K. Brand, Performance of the New RapidFire System for Therapeutic Monitoring of Immunosuppressants, Ther. Drug Monit. 37 (2015) 400-404. doi:10.1097/FTD.0000000000000139.

[7] W. Li, F.L.S. Tse, Special Issue: Review Dried blood spot sampling in combination with LCMS/MS for quantitative analysis of small molecules, Biomed. Chromatogr. 24 (2010) 4965. doi:10.1002/bmc.1367.

[8] P. Denniff, N. Spooner, Volumetric Absorbtive Micro Sampling (VAMS): A Novel Dried Sample Collection Technique for Quantitative Bioanalysis, Anal. Chem. 86 (2014) 84898495. doi:10.1021/ac5022562.

[9] M. Wagner, D. Tonoli, E. Varesio, G. Hopfgartner, The use of mass spectrometry to analyze dried blood spots, Mass Spectrom. Rev. 35 (2016) 361-368. doi:10.1002/mas.21441.

[10] M. Abdel-Rehim, Recent advances in microextraction by packed sorbent for bioanalysis, J. Chromatogr. A. 1217 (2010) 2569-2580. doi:10.1016/j.chroma.2009.09.053. 
[11] É. a. Souza-Silva, N. Reyes-Garcés, G. a. Gómez-Ríos, E. Boyacı, B. Bojko, J. Pawliszyn, E. Boyaci, B. Bojko, J. Pawliszyn, A critical review of the state of the art of solid-phase microextraction of complex matrices iii. bioanalytical and clinical applications, TrAC Trends Anal. Chem. 71 (2015) 249-264. doi:10.1016/j.trac.2015.04.017.

[12] D.S. Jain, G. Subbaiah, M. Sanyal, U.C. Pande, P. Shrivastav, Liquid chromatographytandem mass spectrometry validated method for the estimation of indapamide in human whole blood, J. Chromatogr. B. 834 (2006) 149-154. doi:10.1016/j.jchromb.2006.02.040.

[13] C. Seger, K. Tentschert, W. Stöggl, A. Griesmacher, S.L. Ramsay, A rapid HPLC-MS/MS method for the simultaneous quantification of cyclosporine A, tacrolimus, sirolimus and everolimus in human blood samples., Nat. Protoc. 4 (2009) 526-534. doi:10.1038/nprot.2009.25.

[14] R.A. Koster, E.C.F. Dijkers, D.R.A. Uges, Robust, High-Throughput LC-MS/MS Method for Therapeutic Drug Monitoring of Cyclosporine, Tacrolimus, Everolimus, and Sirolimus in Whole Blood, Ther. Drug Monit. 31 (2009) 116-125. doi:10.1097/FTD.0b013e318192304c.

[15] A. Thomas, H. Geyer, S. Guddat, W. Schänzer, M. Thevis, Dried blood spots (DBS) for doping control analysis, Drug Test. Anal. 3 (2011) 806-813. doi:10.1002/dta.342.

[16] J. Déglon, A. Thomas, P. Mangin, C. Staub, Direct analysis of dried blood spots coupled with mass spectrometry: concepts and biomedical applications., Anal. Bioanal. Chem. 402 (2012) 2485-98. doi:10.1007/s00216-011-5161-6. 
[17] A. Thomas, H. Geyer, W. Schänzer, C. Crone, M. Kellmann, T. Moehring, M. Thevis, Sensitive determination of prohibited drugs in dried blood spots (DBS) for doping controls by means of a benchtop quadrupole/Orbitrap mass spectrometer., Anal. Bioanal. Chem. 403 (2012) 1279-89. doi:10.1007/s00216-011-5655-2.

[18] Q.C. Ji, G. Liu, C.J. D'Arienzo, T. V Olah, M.E. Arnold, What is next for dried blood spots?, Bioanalysis. 4 (2012) 2059-65. doi:10.4155/bio.12.168.

[19] R. Verplaetse, J. Henion, Hematocrit-Independent Quantitation of Stimulants in Dried Blood Spots: Pipet versus Microfluidic-Based Volumetric Sampling Coupled with Automated Flow-Through Desorption and Online Solid Phase Extraction-LC-MS/MS Bioanalysis, Anal. Chem. 88 (2016) 6789-6796. doi:10.1021/acs.analchem.6b01190.

[20] P. Abu-Rabie, P. Denniff, N. Spooner, B.Z. Chowdhry, F.S. Pullen, Investigation of different approaches to incorporating internal standard in DBS quantitative bioanalytical workflows and their effect on nullifying hematocrit-based assay bias, Anal. Chem. 87 (2015) 4996-5003. doi:10.1021/acs.analchem.5b00908.

[21] P. Abu-Rabie, N. Spooner, B.Z. Chowdhry, F.S. Pullen, Optimization of an automated IS addition system for use in high-throughput quantitative DBS analysis, Bioanalysis. 7 (2015) 2763-2775. doi:10.4155/bio.15.196.

[22] P.M.M. De Kesel, W.E. Lambert, C.P. Stove, Does volumetric absorptive microsampling eliminate the hematocrit bias for caffeine and paraxanthine in dried blood samples? A comparative study, Anal. Chim. Acta. 881 (2015) 65-73. doi:10.1016/j.aca.2015.04.056. 
[23] N. Spooner, P. Denniff, L. Michielsen, R. De Vries, Q.C. Ji, M.E. Arnold, K. Woods, E.J. Woolf, Y. Xu, V. Boutet, P. Zane, S. Kushon, J.B. Rudge, A device for dried blood microsampling in quantitative bioanalysis: overcoming the issues associated blood hematocrit, Bioanalysis. 7 (2015) 653-659. doi:10.4155/bio.14.310.

[24] N. Reyes-Garcés, B. Bojko, J. Pawliszyn, High throughput quantification of prohibited substances in plasma using thin film solid phase microextraction, J. Chromatogr. A. 1374 (2014) 40-49. doi:10.1016/j.chroma.2014.11.047.

[25] N. Reyes-Garcés, B. Bojko, D. Hein, J. Pawliszyn, Solid Phase Microextraction Devices Prepared on Plastic Support as Potential Single-Use Samplers for Bioanalytical Applications, Anal. Chem. 87 (2015) 9722-9730. doi:10.1021/acs.analchem.5b01849.

[26] G.A. Gómez-Ríos, J. Pawliszyn, Development of coated blade spray ionization mass spectrometry for the quantitation of target analytes present in complex matrices., Angew. Chem. Int. Ed. Engl. 53 (2014) 14503-7. doi:10.1002/anie.201407057.

[27] G.A. Gómez-Ríos, N. Reyes-Garcés, B. Bojko, J. Pawliszyn, Biocompatible Solid-Phase Microextraction Nanoelectrospray Ionization: An Unexploited Tool in Bioanalysis, Anal. Chem. 88 (2016) 1259-1265. doi:10.1021/acs.analchem.5b03668.

[28] H. Piri-Moghadam, F. Ahmadi, G.A. Gómez-Ríos, E. Boyaci, N. Reyes-Garcés, A. Aghakhani, B. Bojko, J. Pawliszyn, Fast Quantitation of Target Analytes in Small Volumes of Complex Samples by Matrix-Compatible Solid-Phase Microextraction Devices, Angew. Chemie - Int. Ed. 55 (2016) 7510-7514. doi:10.1002/anie.201601476. 
[29] J. Chung, I. De Lannoy, B. Gien, D. Vuckovic, Y. Yang, B. Bojko, J. Pawliszyn, Semiautomated in vivo solid-phase microextraction sampling and the diffusion-based interface calibration model to determine the pharmacokinetics of methoxyfenoterol and fenoterol in rats, Anal. Chim. Acta. 742 (2012) 37-44. doi:10.1016/j.aca.2012.01.034.

[30] D. Vuckovic, S. Risticevic, J. Pawliszyn, In vivo solid-phase microextraction in metabolomics: opportunities for the direct investigation of biological systems., Angew. Chem. Int. Ed. Engl. 50 (2011) 5618-28. doi:10.1002/anie.201006896.

[31] D. Vuckovic, I. de Lannoy, B. Gien, R.E. Shirey, L.M. Sidisky, S. Dutta, J. Pawliszyn, In vivo solid-phase microextraction: capturing the elusive portion of metabolome., Angew. Chem. Int. Ed. Engl. 50 (2011) 5344-8. doi:10.1002/anie.201006715.

[32] D. Vuckovic, I. De Lannoy, B. Gien, Y. Yang, F.M. Musteata, R. Shirey, L. Sidisky, J. Pawliszyn, In vivo solid-phase microextraction for single rodent pharmacokinetics studies of carbamazepine and carbamazepine-10,11-epoxide in mice., J. Chromatogr. A. 1218 (2011) 3367-3375. doi:10.1016/j.chroma.2010.07.060.

[33] X. Zhang, A. Es-Haghi, J. Cai, J. Pawliszyn, Simplified kinetic calibration of solid-phase microextraction for in vivo pharmacokinetics., J. Chromatogr. A. 1216 (2009) 7664-9. doi:10.1016/j.chroma.2009.09.021.

[34] F.M. Musteata, I. de Lannoy, B. Gien, J. Pawliszyn, Blood sampling without blood draws for in vivo pharmacokinetic studies in rats., J. Pharm. Biomed. Anal. 47 (2008) 907-12. doi:10.1016/j.jpba.2008.03.028. 
[35] X. Zhang, A. Es-haghi, F.M. Musteata, G. Ouyang, J. Pawliszyn, Quantitative in vivo microsampling for pharmacokinetic studies based on an integrated solid-phase microextraction system, Anal. Chem. 79 (2007) 4507-4513. doi:10.1021/ac070177c.

[36] F.M. Musteata, M.L. Musteata, J. Pawliszyn, Fast in vivo microextraction: A new tool for clinical analysis, Clin. Chem. 52 (2006) 708-715. doi:10.1373/clinchem.2005.064758.

[37] B. Bojko, E. Cudjoe, G. a Gómez-Ríos, K. Gorynski, R. Jiang, N. Reyes-Garcés, S. Risticevic, É. a S. Silva, O. Togunde, D. Vuckovic, J. Pawliszyn, SPME--quo vadis?, Anal. Chim. Acta. 750 (2012) 132-51. doi:10.1016/j.aca.2012.06.052.

[38] P.H. Hinderling, Red blood cells: a neglected compartment in pharmacokinetics and pharmacodynamics., Pharmacol. Rev. 49 (1997) 279-295.

file:///Users/Aniradha/Desktop/Dropbox/Papers2/Library.papers3/Files/2C/2C76C202E9DF-4726-A8D5-E3499CC2D344.pdf\%5Cnpapers3://publication/uuid/000db765-aa91415b-a07d-47bc09a39c86.

[39] M.L. Musteata, F.M. Musteata, J. Pawliszyn, Biocompatible solid-phase microextraction coatings based on polyacrylonitrile and solid-phase extraction phases., Anal. Chem. 79 (2007) 6903-6911.

[40] A. Tan, S. Gagné, I.A. Lévesque, S. Lachance, N. Boudreau, A. Lévesque, Impact of hemolysis during sample collection: How different is drug concentration in hemolyzed plasma from that of normal plasma?, J. Chromatogr. B. 901 (2012) 79-84. doi:10.1016/j.jchromb.2012.06.002. 
[41] B.K. Matuszewski, M.L. Constanzer, C.M. Chavez-Eng, Strategies for the assessment of matrix effect in quantitative bioanalytical methods based on HPLC-MS/MS., Anal. Chem. 75 (2003) 3019-30. http://www.ncbi.nlm.nih.gov/pubmed/12964746.

[42] M.N. Alam, L. Ricardez-Sandoval, J. Pawliszyn, Calibrant Free Sampling and Enrichment with Solid-Phase Microextraction: Computational Simulation and Experimental Verification, Ind. Eng. Chem. Res. 56 (2017) 3679-3686. doi:10.1021/acs.iecr.7b00131.

[43] S.S. Mohammed, M.M. Christopher, P. Mehta, A. Kedar, S. Gross, H. Derendorf, Increased Erythrocyte and Protein Binding of Codeine in Patients with Sickle Cell Disease, J. Pharm. Sci. 82 (1993) 1112-1117. doi:10.1002/jps.2600821109.

[44] A.R. Pries, D. Neuhaus, P. Gaehtgens, Blood viscosity in tube flow: dependence on diameter and hematocrit., Am. J. Physiol. 263 (1992) H1770-8. http://www.ncbi.nlm.nih.gov/pubmed/1481902 (accessed June 6, 2017).

[45] G. Skopp, L. Pötsch, M. Mauden, B. Richter, Partition coefficient, blood to plasma ratio, protein binding and short-term stability of 11-nor- $\Delta 9$-carboxy tetrahydrocannabinol glucuronide, Forensic Sci. Int. 126 (2002) 17-23. doi:10.1016/S0379-0738(02)00023-3.

[46] M.A. Huestis, Estimating the Time of Last Cannabis Use from Plasma 9Tetrahydrocannabinol and 11-nor-9-Carboxy-9-Tetrahydrocannabinol Concentrations, Clin. Chem. 51 (2005) 2289-2295. doi:10.1373/clinchem.2005.056838.

[47] E. Boyacı, K. Gorynski, A. Rodriguez-Lafuente, B. Bojko, J. Pawliszyn, Introduction of solidphase microextraction as a high-throughput sample preparation tool in laboratory 
analysis of prohibited substances, Anal. Chim. Acta. 809 (2014) 69-81.

http://www.sciencedirect.com/science/article/pii/S0003267013015110 (accessed January 13, 2014).

[48] ChemSpider | Search and share chemistry, (2015). http://www.chemspider.com/?gclid=CjOKEQjwOtCuBRDljJ_Mlb6zzpQBEiQAyjCoBuRuclLsj hkbZ_HWuKBN5uhjex7TiSjf_30nthgViMwaAh1M8P8HAQ (accessed August 19, 2015).

[49] DrugBank, (n.d.). http://www.drugbank.ca/ (accessed July 6, 2014).

[50] R.S. Obach, F. Lombardo, N.J. Waters, Trend analysis of a database of intravenous pharmacokinetic parameters in humans for 670 drug compounds, Drug Metab. Dispos. 36 (2008) 1385-1405. doi:10.1124/dmd.108.020479.

[51] I. Yamamoto, K. Iwata, M. Nakashima, Pharmacokinetics of plasma and urine clenbuterol in man, rat, and rabbit., J. Pharmacobiodyn. 8 (1985) 385-91.

http://www.ncbi.nlm.nih.gov/pubmed/4045696 (accessed May 23, 2017). 
Table 1. Model compounds with their corresponding physicochemical properties and MS/MS detection parameters. Analytes are listed in order of hydrophobicity.

\begin{tabular}{|c|c|c|c|}
\hline $\begin{array}{c}\text { Compound } \\
\text { SRM transition } \\
\text { Collision energy, S-lenses }\end{array}$ & $\begin{array}{c}\text { Structure (molecular weight, } \\
\text { MW) }\end{array}$ & $\log P[48]$ & $\begin{array}{l}\text { Plasma protein } \\
\text { binding, \%[49] }\end{array}$ \\
\hline $\begin{array}{c}\text { Nikethamide } \\
179.10 \rightarrow 108.10 \\
18,76\end{array}$ & MW: 178.23 & 0.33 & NA \\
\hline $\begin{array}{c}\text { Salbutamol } \\
240.14 \rightarrow 148.10 \\
18,59\end{array}$ & MW: 239.31 & 0.64 & $8[50]$ \\
\hline $\begin{array}{c}\text { Codeine } \\
300.11 \rightarrow 165.10 \\
64,104\end{array}$ & MW: 299.36 & 1.19 & $7-25$ \\
\hline $\begin{array}{c}\text { Metoprolol } \\
268.14 \rightarrow 116.15 \\
18,94\end{array}$ & $\mathrm{H}_{3} \mathrm{CO}^{-}$ & 1.88 & 12 \\
\hline $\begin{array}{c}\text { Methamphetamine } \\
\begin{array}{c}150.11 \rightarrow 91.12 \\
19,45\end{array}\end{array}$ & 24 & 2.07 & $10-20$ \\
\hline $\begin{array}{c}\text { Clenbuterol } \\
277.068 \rightarrow 203.049 \\
15,70\end{array}$ & $\mathrm{MW}: 276.08$ & 2.61 & 89 - 98[51] \\
\hline $\begin{array}{c}\text { Exemestane } \\
297.173 \rightarrow 121.118 \\
19,72\end{array}$ & MW: 296.18 & 3.11 & 90 \\
\hline
\end{tabular}




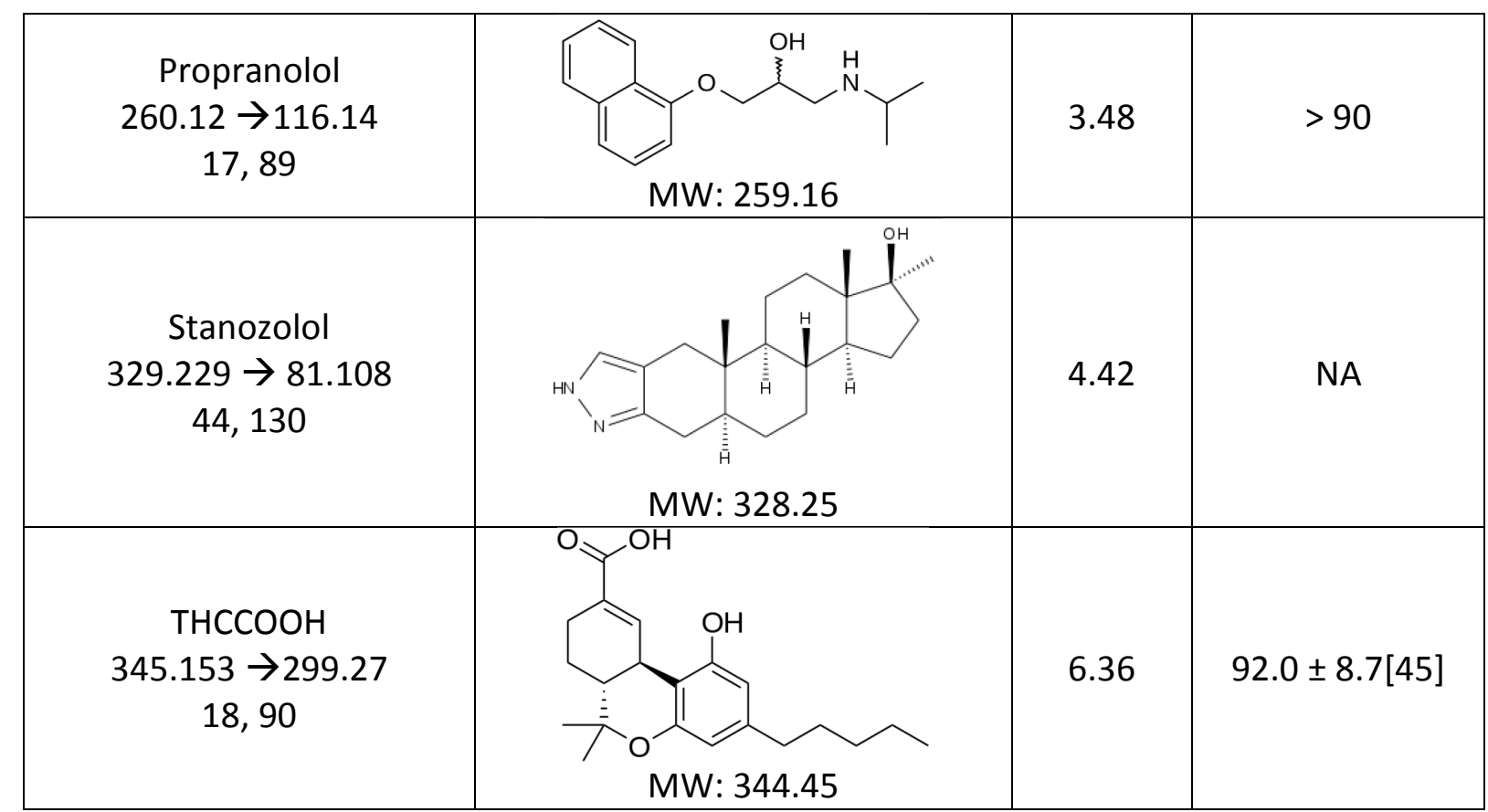

$\mathrm{NA}=$ not available 
Table 2. Slopes of the calibration curves constructed using blood samples with different Hct levels spiked with drugs at concentrations in the range between 5 and $100 \mathrm{ng} \mathrm{mL}^{-1}$ (no internal standard).

\begin{tabular}{|c|c|c|c|c|c|c|c|c|c|c|}
\hline \multirow{3}{*}{ Compound (case) } & \multicolumn{10}{|c|}{ Slopes $(n=4)^{a}$ (average \pm standard deviation) } \\
\hline & \multicolumn{5}{|c|}{ MM-F, 400 rpm } & \multicolumn{5}{|c|}{ HLB-D, 1500 rpm } \\
\hline & $20 \% \mathrm{Hct}$ & $45 \% \mathrm{Hct}$ & $70 \% \mathrm{Hct}$ & RSD \% ${ }^{b}$ & $\mathbf{F}^{\mathrm{c}}$ & $20 \% \mathrm{Hct}$ & $45 \% \mathrm{Hct}$ & $70 \% \mathrm{Hct}$ & RSD \% ${ }^{b}$ & $F^{\mathrm{c}}$ \\
\hline Salbutamol (I) & $0.018 \pm 0.003$ & $0.016 \pm 0.006$ & $0.017 \pm 0.004$ & 4.0 & 0.090 & $0.25 \pm 0.02$ & $0.24 \pm 0.04$ & $0.23 \pm 0.02$ & 3.0 & 0.230 \\
\hline Nikethamide (I) & $0.042 \pm 0.004$ & $0.044 \pm 0.004$ & $0.039 \pm 0.004$ & 6.6 & 0.017 & $0.71 \pm 0.06$ & $0.73 \pm 0.11$ & $0.75 \pm 0.06$ & 3.0 & 0.238 \\
\hline Methamphetamine (I) & $0.058 \pm 0.005$ & $0.053 \pm 0.009$ & $0.048 \pm 0.003$ & 9.5 & 2.865 & $0.55 \pm 0.04$ & $0.54 \pm 0.07$ & $0.54 \pm 0.04$ & 1.0 & 0.022 \\
\hline Codeine (I) & $0.034 \pm 0.002$ & $0.030 \pm 0.004$ & $0.029 \pm 0.002$ & 8.0 & 3.090 & $0.69 \pm 0.06$ & $0.69 \pm 0.05$ & $0.69 \pm 0.07$ & 0.1 & $3.41 \mathrm{e}^{-4}$ \\
\hline Propranolol (II) & $0.047 \pm 0.002$ & $0.036 \pm 0.003$ & $0.027 \pm 0.003$ & 26.0 & 61.138 & $0.56 \pm 0.05$ & $0.57 \pm 0.06$ & $0.58 \pm 0.04$ & 2.0 & 0.232 \\
\hline Metoprolol (II) & $0.077 \pm 0.008$ & $0.064 \pm 0.008$ & $0.053 \pm 0.006$ & 19.0 & 11.108 & $0.83 \pm 0.08$ & $0.85 \pm 0.10$ & $0.88 \pm 0.08$ & 3.0 & 0.243 \\
\hline Clenbuterol (II) & $0.072 \pm 0.004$ & $0.059 \pm 0.005$ & $0.045 \pm 0.004$ & 14.0 & 36.232 & $0.69 \pm 0.04$ & $0.70 \pm 0.04$ & $0.73 \pm 0.05$ & 3.0 & 1.031 \\
\hline Exemestane (III) & $0.034 \pm 0.005$ & $0.023 \pm 0.001$ & $0.013 \pm 0.003$ & 45.0 & 37.719 & $0.31 \pm 0.05$ & $0.28 \pm 0.03$ & $0.21 \pm 0.03$ & 20.0 & 7.597 \\
\hline $\left.\mathrm{THCCOOH}_{(\mathrm{IV}}\right)^{\mathrm{d}}$ & $0.010 \pm 0.002$ & $0.011 \pm 0.001$ & $0.012 \pm 0.001$ & 9.6 & 1.370 & $0.026 \pm 0.001$ & $0.032 \pm 0.006$ & $0.036 \pm 0.002$ & 16.2 & 8.851 \\
\hline Stanozolol (IV) & $0.040 \pm 0.006$ & $0.036 \pm 0.003$ & $0.034 \pm 0.004$ & 7.7 & 1.539 & $0.047 \pm 0.004$ & $0.053 \pm 0.006$ & $0.066 \pm 0.007$ & 18.0 & 11.865 \\
\hline
\end{tabular}

${ }^{a}$ Average slopes calculated from the equation $y=m x+b$, where $y$ is the amount extracted in $n g$ and $x$ is the concentration spiked in blood.

${ }^{b}$ inter-slope RSD estimated from the values obtained for each Hct level.

${ }^{\mathrm{C}} \mathrm{F}$ crit $=4.256(\alpha=0.05)$, if $\mathrm{F}>\mathrm{F}$ crit the null hypothesis that the three slopes are equal is rejected (one-way ANOVA).

${ }^{\mathrm{d}}$ The lowest concentration level was $25 \mathrm{ng} \mathrm{mL}^{-1}$. 
Table 3. Corrected calibration curve slopes for those cases where F>Fcrit.

\begin{tabular}{|c|c|c|c|c|c|}
\hline \multirow{2}{*}{\begin{tabular}{c} 
Compound (internal standard) \\
\cline { 2 - 6 }
\end{tabular}} & \multicolumn{5}{|c|}{ Slopes (n=4) (average \pm standard deviation) } \\
\cline { 2 - 6 } & $\mathbf{2 0 \% ~ H c t ~}$ & $\mathbf{4 5} \%$ Hct & $\mathbf{7 0 \% ~ H c t ~}$ & RSD \% & F $^{\text {b }}$ \\
\hline $\begin{array}{c}\text { Propranolol, MM-F } \\
\text { (Methadone-d3) }\end{array}$ & $0.018 \pm 0.002$ & $0.019 \pm 0.001$ & $0.017 \pm 0.001$ & 5.4 & 2.538 \\
\hline $\begin{array}{c}\text { Metoprolol, MM-F } \\
\text { (Methadone-d3) }\end{array}$ & $0.0053 \pm 0.0002$ & $0.0054 \pm 0.0002$ & $0.0055 \pm 0.0003$ & 1.2 & 0.331 \\
\hline $\begin{array}{c}\text { Clenbuterol, MM-F } \\
\text { (Methadone-d3) }\end{array}$ & $0.024 \pm 0.001$ & $0.024 \pm 0.001$ & $0.023 \pm 0.001$ & 3.1 & 3.058 \\
\hline $\begin{array}{c}\text { THCCOOH }{ }^{b}, \mathrm{HLB}-\mathrm{D} \\
\text { (THCCOOH-d3) }\end{array}$ & $0.011 \pm 0.001$ & $0.011 \pm 0.001$ & $0.012 \pm 0.001$ & 5.8 & 3.872 \\
\hline $\begin{array}{c}\text { Stanozolol, HLB-D } \\
\text { (THCCOOH-d3) }\end{array}$ & $0.41 \pm 0.06$ & $0.42 \pm 0.02$ & $0.46 \pm 0.03$ & 5.9 & 1.653 \\
\hline
\end{tabular}

${ }^{a}$ Average slopes calculated from the equation $y=m x+b$, where $y$ is the ratio area analyte/area internal standard and $x$ is the concentration spiked in blood.

${ }^{\mathrm{b}} \mathrm{F}$ crit $=4.256(\alpha=0.05)$, if $\mathrm{F}>\mathrm{F}$ crit the null hypothesis that the three slopes are equal is rejected (one-way ANOVA).

'The lowest concentration level was $25 \mathrm{ng} \mathrm{mL}^{-1}$. 


\section{Figures captions}

Figure 1. Extraction time profiles obtained at different experimental conditions for four representative analytes: salbutamol ( $a$ and b), propranolol ( $c$ and $d$ ), exemestane (e and f), $\mathrm{THCCOOH}$ ( $\mathrm{g}$ and $\mathrm{h}$ ). Plots on the left side summarize results for MM-F at $400 \mathrm{rpm}$, and plots on the right side summarize results for HLB-D at $1500 \mathrm{rpm}$.

Figure 2. Relative recoveries obtained after extracting from $70 \% \mathrm{Hct}$ blood samples (hemolyzed vs. non-hemolyzed) spiked at $100 \mathrm{ng} \mathrm{mL}^{-1}$. These experiments were conducted using HLB-D at $1500 \mathrm{rpm}$ and an extraction time of $90 \mathrm{~min}$.

Figure 3. Absolute matrix effects assessed in $70 \%$ Hct blood samples (hemolyzed and nonhemolyzed). Extracts from blank blood samples obtained with HLB-D (90 min extraction at 1500 $\mathrm{rpm}$ ) were spiked with the model compounds at $50 \mathrm{ng} \mathrm{mL}^{-1}$, and their response was compared with the one from standards spiked in neat solvent at the same concentration level. Acceptable absolute matrix effect range is represented in dotted lines (80 to 120\%). 
Figure 1
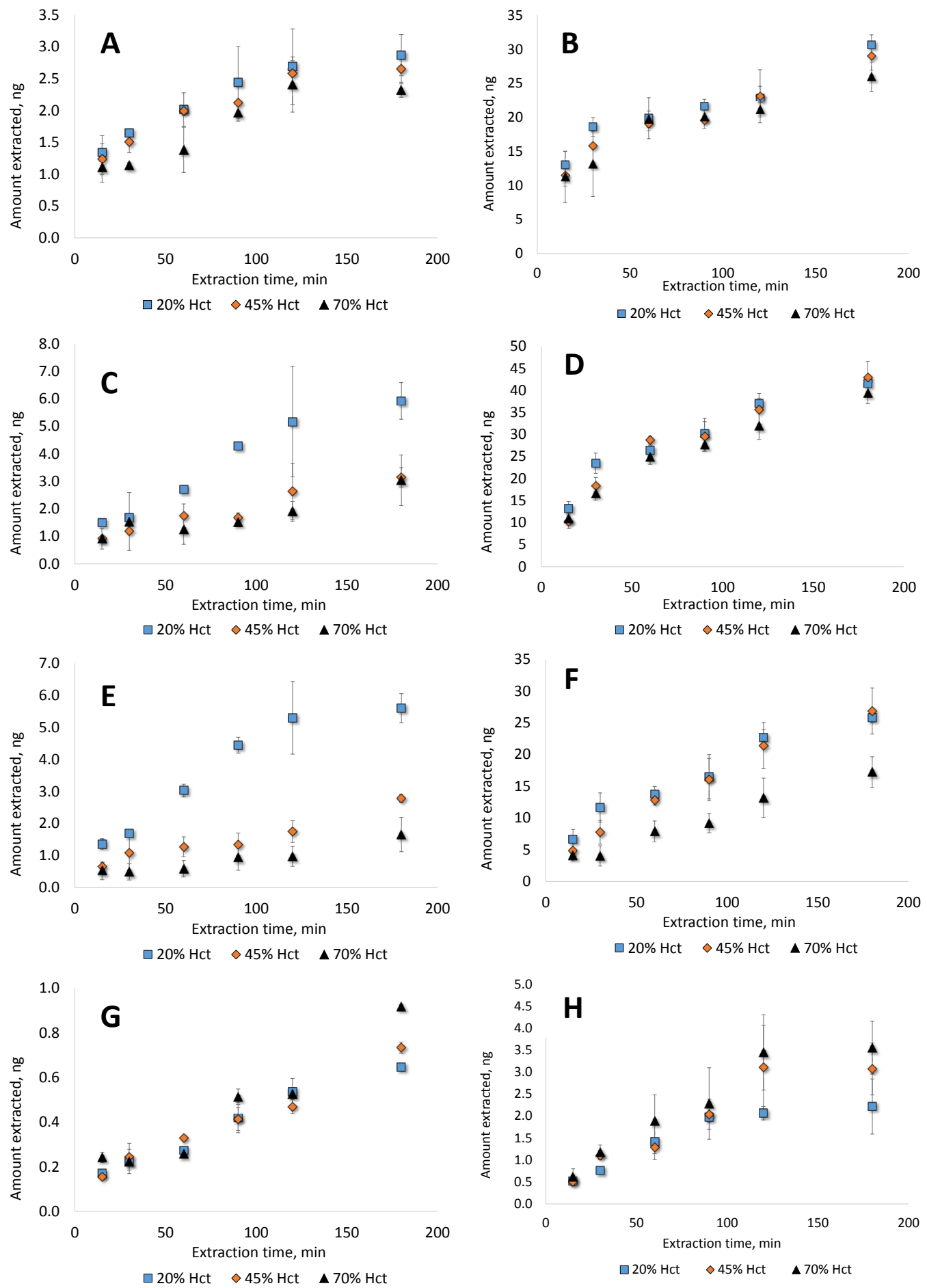
Figure 2

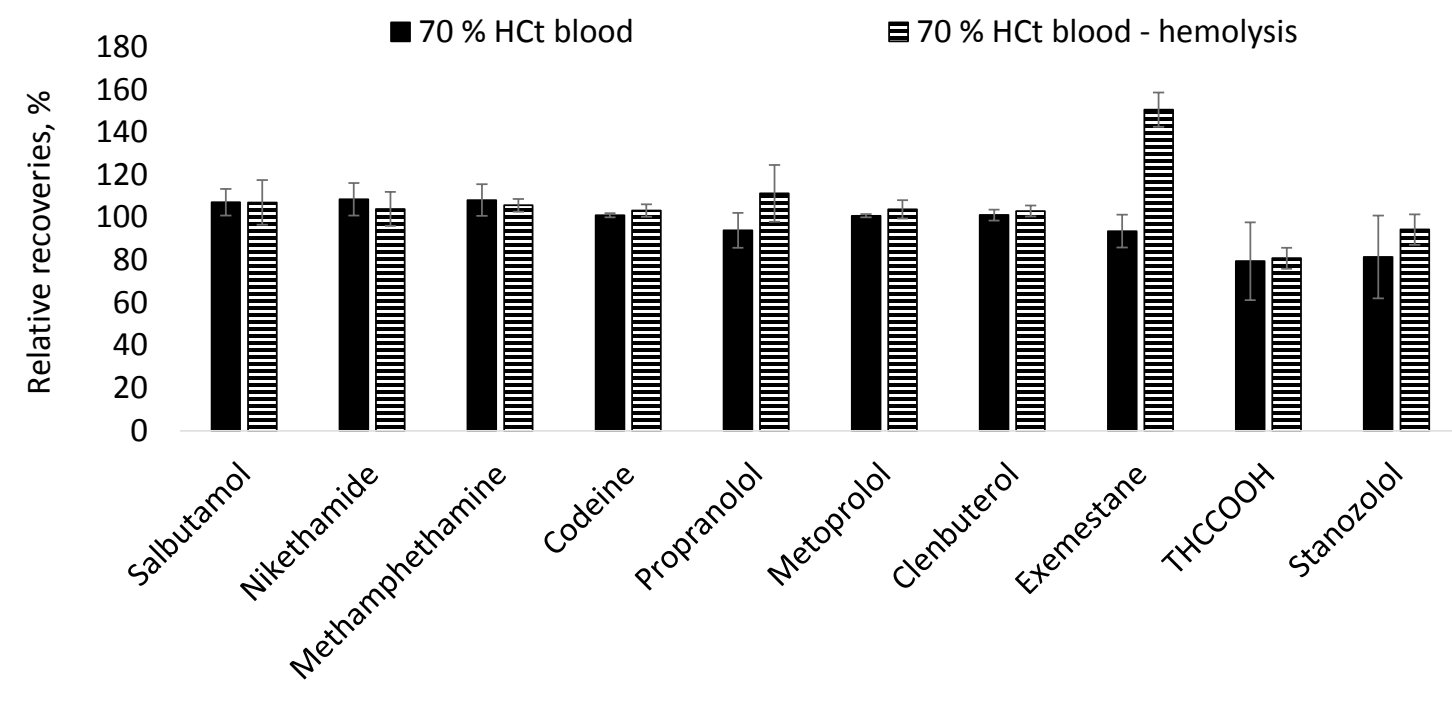




\section{Figure 3}

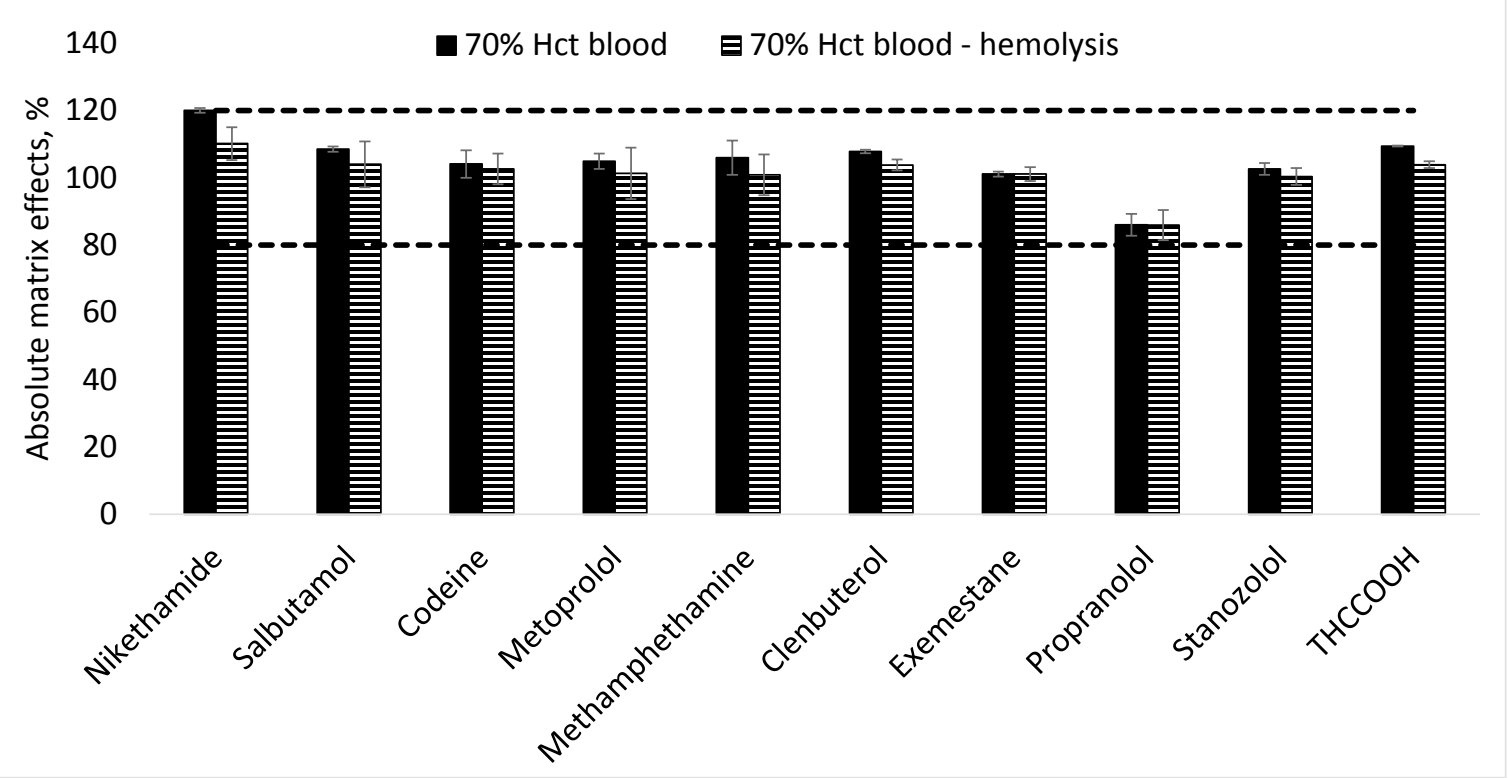




\section{Graphical abstract}
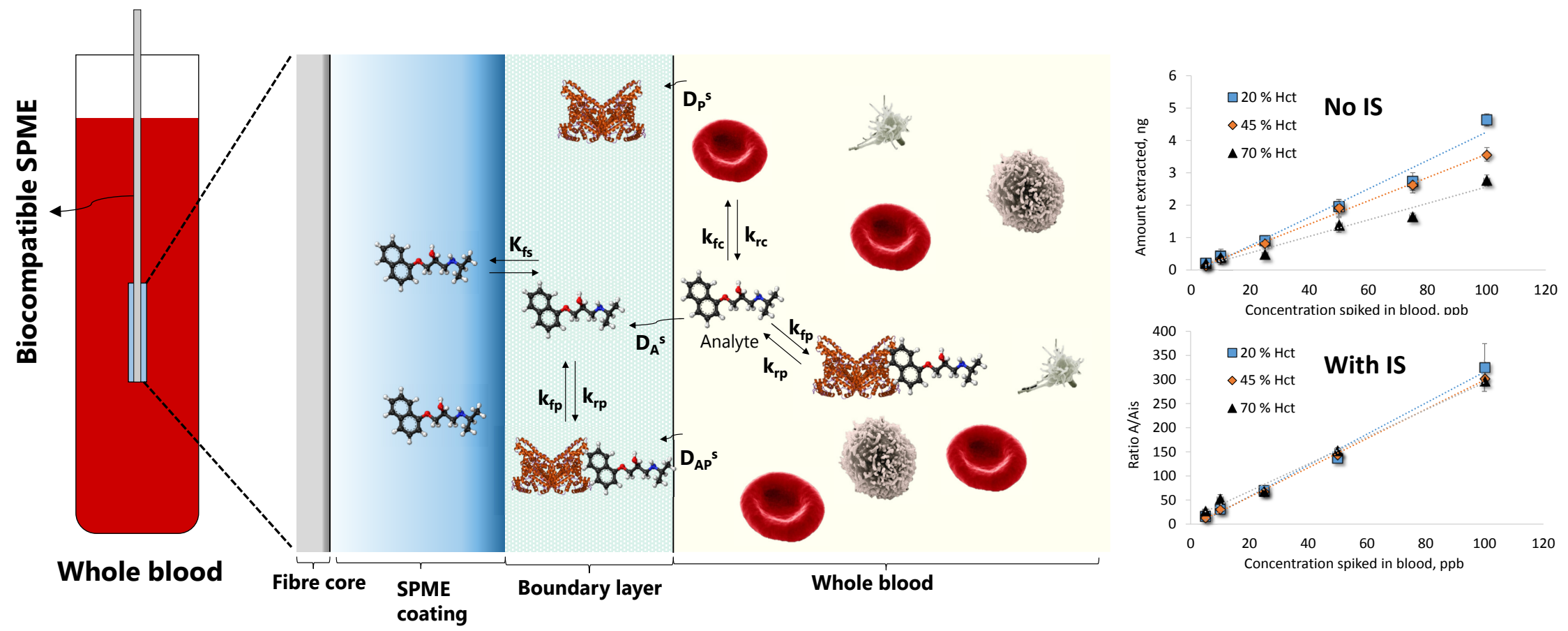
